\title{
Elevated Thyroid Indices in Children and Adolescents with Obsessive-Compulsive Disorder: Effects of Clomipramine Treatment
}

\author{
James T. McCracken, M.D., ${ }^{1,2}$ and Gregory L. Hanna, M.D. ${ }^{3}$
}

\begin{abstract}
Objective: To examine the basal thyroid function in pediatric Obsessive Compulsive Disorder (OCD) versus controls, and to explore the relation between baseline thyroid measures and response to clomipramine treatment, and the effects of treatment on thyroid hormones.

Methods: Sixteen children and adolescents with DSM-III-R OCE and 13 control children and adolescents without psychiatric illness were compared on basal measures of thyroidstimulating hormone (TSH), triiodothyronine (T3), and thyroxine (T4). For the OCD subjects, samples were compared pre- and post- 4 weeks of treatment with clomipramine. Response of OCD symptoms was measured by the Children's Yale-Brown Obsessive Compulsive Scale (CY-BOCS).

Results: OCD subjects demonstrated subtle but significant elevations of TSH, T3, and T4 pre-treatment compared to controls. Clomipramine treatment was associated with significant decreases in TSH and T3 concentrations. Pre-treatment TSH and T4 concentrations correlated with reductions in CY-BOCS following 8 weeks of clomipramine.

Conclusion: Elevated thyroid function at baseline may be a biomarker of OCD improvement, and may reflect aspects of the underlying pathophysiology of OCD.
\end{abstract}

\section{INTRODUCTION}

$\mathrm{O}$ BSESSIVE-COMPULSIVE DISORDER (OCD) is a chronic, severe neuropsychiatric disorder with relatively early onset, including for many, onset of symptoms occurring in childhood or adolescence (Noshirvani et al. 1991). The frequent association of OCD with symptoms of depression and anxiety, and responsiveness to selected antidepressants has led many investigators to examine for possible links between
OCD and a diverse group of depression-related biological abnormalities. These include abnormalities suggested to reflect the pathophysiology of depression and anxiety, for example, sleep EEG correlates such as shortened REM latency, platelet receptor differences, and hormonal differences, including differences between adult OCD patients and controls in the regulation of the hypothalamo-pituitarythyroid (HPT) axis. Overall however, thyroid function in OCD has received scant attention.

\footnotetext{
1UCLA Neuropsychiatric Institute, Los Angeles, California. ${ }^{2}$ Mental Retardation Research Center, Los Angeles, California. ${ }^{3}$ Department of Psychiatry, University of Michigan Medical Center, Ann Arbor, Michigan.
} 
The two available studies do suggest that HPT function may be altered in patients with OCD.

There are several outstanding questions relating to HPT function and OCD. First, baseline thyroid function has not been well characterized in OCD patients. To our knowledge, only two studies have reported detailed information on thyroid function tests in OCD patients (Joffe and Swinson 1988; Aizenberg et al. 1991). These two reports have suggested normal basal thyroid function tests in adults with OCD, but one was limited by their use of depressed adults as the only comparison group (Joffe and Swinson 1988). However in the Joffe and Swinson (1988) study, while no differences in mean triiodothyronine (T3) or thyroxine (T4) levels were observed, mean thyroxine (TSH) was elevated in the OCD patients versus depressives (though skewed by one patient) with large variability noted. In the second report, no differences in $\mathrm{T} 3, \mathrm{~T} 4$, or baseline TSH were found for 10 adults with OCD versus 10 age- and sex-matched normal controls, but a significant blunting of the TSH response to thyrotropin releasing hormone (TRH) was noted for the OCD subjects, with $70 \%$ manifesting a blunted response by virtue of their having a DTSH of $<5 \mathrm{mu} / \mathrm{ml}$ (Aizenberg et al. 1991). Therefore, thyroid function abnormalities in this small number of OCD patients were not uncommon. One additional criticism of the extant data on basal thyroid status is that both investigations relied upon the older double antibody radioimmunoassay method for TSH measurement. Because daytime TSH concentrations are quite low, it is possible that subtle patient-control differences have been obscured by a "floor" effect. The advent of the new generation of ultrasensitive immunoradiometric (IRMA) methods for TSH enables a more accurate comparison of basal TSH concentrations between OCD patients and comparison groups.

Second, few biological studies of OCD have focused on children and adolescents with OCD, and to our knowledge no studies of juvenile OCD subjects have reported on thyroid status. For biological studies, younger OCD patients have the theoretical advantages of having received little or no prior pharmacologic treatment, are less likely to have experi- enced secondary psychiatric illnesses or physical illnesses that may have biological sequelae, and reduce the influence of aging per se on biological functions.

Lastly, the effects of pharmacologic treatment for OCD on thyroid function and the influence of initial thyroid function on response to treatment have not been examined. One controlled trial of adjunctive T3 versus lithium administration to a group of adult OCD subjects with a "partial" response to clomipramine treatment was reportedly without effect, although lithium was noted to reduce depressive symptoms (Pigott et al. 1991). As mentioned above, it is not established if OCD patients are more likely to possess subtle hypo- or hyperthyroidism, leaving the rationale for adjunctive T3 administration unclear.

The purpose of the present report was to further our understanding of the relation between the HPT axis and OCD by examining basal HPT measures in a population of children and adolescents with OCD versus agematched normal controls, and to assess the influence of 4 weeks of clomipramine treatment on HPT measures.

\section{MATERIALS AND METHODS}

\section{Subjects}

Portions of the study design and clinical response to clomipramine treatment have been described previously (Hanna et al. 1991). Sixteen subjects (twelve males, four females) with OCD diagnosed by DSM-III-R criteria (APA 1987) and thirteen normal control children and adolescents (seven males, six females) without a personal lifetime history of any Axis I psychiatric disorder comprised the study sample. Ages ranged from nine to seventeen years (mean $=13.6$ years) for the OCD subjects. Normal control subjects ranged in age from ten to seventeen years (mean=13.1 years). All study participants underwent a comprehensive diagnostic assessment consisting of a clinical interview, a structured research diagnostic interview, and completion of rating scales, which included (for the OCD subjects only) the Children's Yale-Brown Obsessive-Compul- 
sive Scale (CY-BOCS; Goodman and Price 1989), and the NIMH Global Obsessive-Compulsive Scale (GOCS; Murphy et al. 1989). All OCD subjects scored $>16$ on the CY-BOCS (Goodman and Price 1989) and at least seven on the GOCS at initial assessment. Subjects meeting criteria for OCD were excluded if any of the following were present: A 17-item Hamilton Depression Rating Score (HDRS) of 16 or more, major medical illness, mental retardation, organic mental disorder, pervasive developmental disorder, psychosis, schizotypal personality disorder, bipolar disorder, panic disorder, agoraphobia, eating disorders, or suicidal behavior. As above, the normal controls were required to be free of any lifetime history of Axis I psychiatric disorders. None of the patients had received any medications during the 14 days prior to initial blood sampling.

Following baseline evaluation, all OCD subjects were treated with clomipramine, which was titrated similarly over three weeks to a maximum daily dose of $3 \mathrm{mg} / \mathrm{kg}$. At four weeks of treatment the mean daily dose was $2.67 \mathrm{mg} / \mathrm{kg}$. Response to treatment was assessed by repeat CY-BOCS administration, and by clinician rating of minimal, moderate, or good response to treatment as determined by clinical change at 8 weeks.

\section{Hormone assays}

For the OCD subjects, afternoon resting blood samples were drawn at baseline and again at four weeks of clomipramine treatment for hormone measurements. Afternoon blood samples from the normal controls were also obtained at rest. Plasma concentrations of total T3 and total T4 were measured in duplicate by coated-tube double antibody radioimmunoassays (IMMUNOCHEM, ICN Biomedicals, Costa Mesa, CA). Plasma was analyzed for TSH by IRMA assay (ICN Biomedicals, Costa Mesa, CA). Assays were performed by laboratory personnel blind to subject status and study design. For T3 and T4, samples were assayed in two separate batches. All samples were assayed in a single batch for TSH. For the TSH assay, the minimal detectable dose is $0.04 \mathrm{mIU} / \mathrm{ml}$, with a normal range of $0.3-6.5 \mathrm{mIU} / \mathrm{ml}$. Coefficients of variation were $4.2,3.3$, and $4.7 \%$ for the low, medium, and high pools, respectively.

\section{Data analysis}

Patient-control comparisons were analyzed by individual unpaired t-tests of independent means. For analysis of the effects of clomipramine treatment, baseline and 4-week data from the OCD subjects were analyzed by paired t-tests. Pearson product-moment correlations were computed for the thyroid measures. Statistical significance was set at $\mathrm{p}<0.05$ for twotailed tests.

\section{RESULTS}

Age and sex compositions of the two samples were not significantly different. Comparison of the OCD subjects before clomipramine treatment to normal controls showed the OCD subjects to demonstrate significantly higher concentrations for all three thyroid measures. The OCD subjects showed significantly greater total T3 concentrations than controls (OCD vs. control, mean + SEM: $156+8.3$ vs $117+5.0 \mathrm{ng} / \mathrm{dl} ; \mathrm{t}=3.86, \mathrm{p}<.0006)$ (Figure 1). Similarly, total T4 concentrations were also greater in the OCD compared to controls $(10.2+0.5$ vs. $7.7+0.34 \mathrm{mg} / \mathrm{dl} ; \mathrm{t}=4.02, \mathrm{p}<$ 0.0004) (Figure 2). TSH was also significantly greater in the OCD subjects compared to controls $(1.49+.18$ vs. $.73+.10 \mathrm{mIU} / \mathrm{ml} ; \mathrm{t}=3.58$; $\mathrm{p}<.002$ ) (Figure 3).

Paired $t$ tests between baseline and four week samples for the OCD subjects revealed a significant effect of clomipramine treatment on T3, with T3 concentrations at 4 weeks significantly lower than baseline concentrations $(156+8.3$ vs. $141+10.3 \mathrm{ng} / \mathrm{dl}, \mathrm{t}=2.23, \mathrm{p}<$ 0.05. Similarly, TSH concentrations were significantly reduced after 4 weeks of treatment $(1.49+.18$ vs. $1.10+.11 \mathrm{mIU} / \mathrm{ml} ; \mathrm{t}=2.68, \mathrm{p}<$ .02). However, paired t-tests failed to show a significant effect of clomipramine on T4 after four weeks of treatment (baseline vs. 4 weeks, $10.2+.5$ vs. $10.1+.11 \mathrm{mg} / \mathrm{dl}$; NS). No significant relations were noted between T3, T4, or TSH hormone concentration and sex, or presence of washing compulsions. 


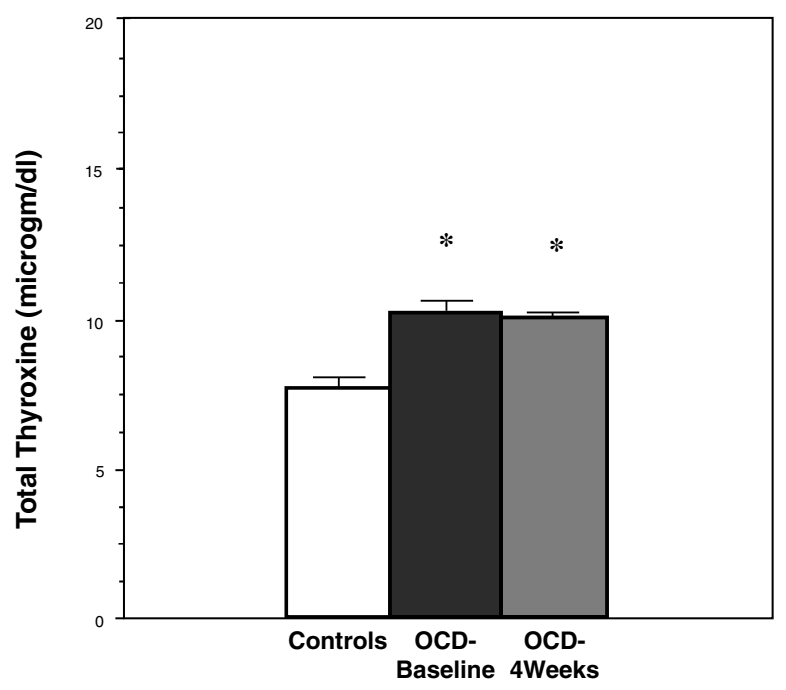

* Significantly different versus controls; $p<0.002$.

FIG. 1.

Because of our interest in thyroid influences on clinical response to clomipramine treatment, we also performed exploratory correlational analyses between baseline thyroid hormone concentrations and symptom measures after eight weeks of clomipramine. Correlational analysis showed that $r=0.70(p<.01$, two-tailed) for baseline T4 versus percent reduction in CY-BOCS from baseline to eight weeks of treatment, and $r=0.56(p<.05$, two-

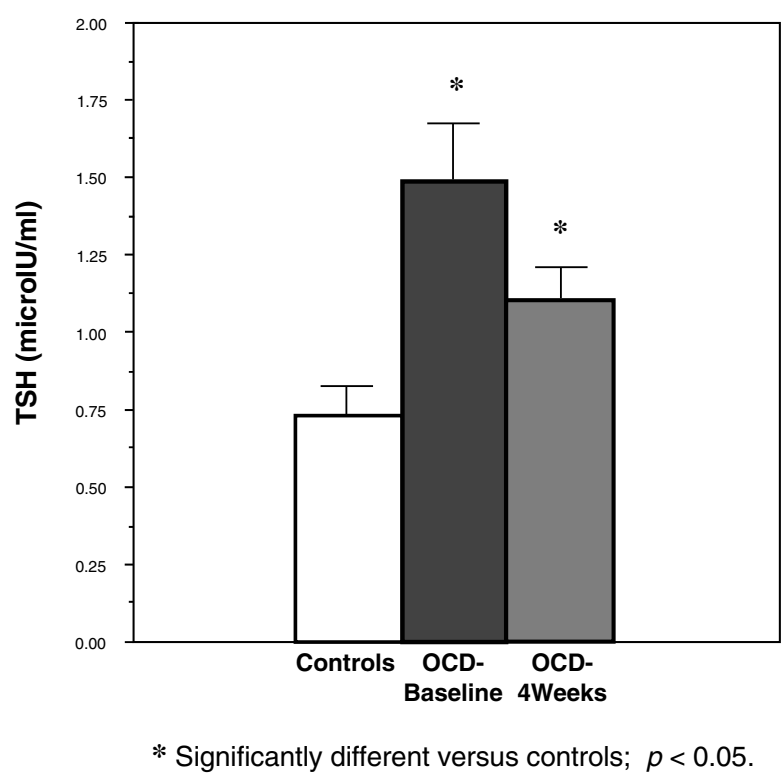

FIG. 2.

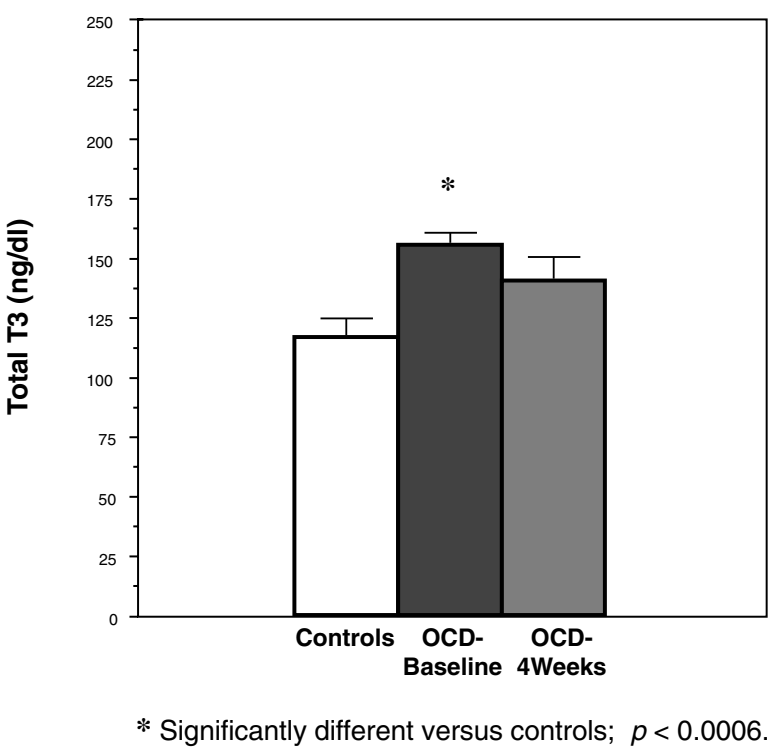

FIG. 3.

tailed) for baseline TSH versus percent reduction in CY-BOCS from baseline to eight weeks of treatment. Other correlations between T3 and symptom and symptom change measures were not significant.

\section{DISCUSSION}

Although limited by sample size, few hormone measures, and a broad age range, several provocative findings of interest emerged from our pilot study. First, untreated outpatient children and adolescents with OCD compared to normal controls appeared to manifest subtle but detectable elevations in basal thyroid function measures, specifically higher $\mathrm{TSH}$, total T3, and total T4 concentrations. Second, 4 weeks of clomipramine administration significantly reduced TSH and T3, but not T4 concentrations. Lastly, and of potential relevance to the treatment of OCD, basal T4 and TSH concentrations before treatment were possibly associated with the extent of symptomatic improvement after 8 weeks of clomipramine treatment.

The findings in our outpatient child and adolescent OCD sample of increased TSH, T3, and $\mathrm{T} 4$ concentrations compared to normal controls are not in agreement with reports of 
normal TSH, T3 and T4 levels in adults with OCD by Joffe and Swinson (1988) and Aizenberg et al. (1991). The reason for such disagreement between our data and prior reports may rest in the younger age of our sample, the absence of any prior pharmacologic treatment in $40 \%$ of our OCD subjects, exclusion of comorbid major depression in our subjects, the timing of sample collection (afternoon versus morning samples in the Aizenberg et al. study), the proportion of females, and assay methods.

Earlier studies of heterogenous samples of hospitalized psychiatric patients have reported elevated free thyroxine index (FTI) values (Cohen and Swigar 1979; Morley and Shafer 1982; Spratt et al. 1982; Caplan et al. 1983; Chopra et al. 1990), elevated T4 (Spratt et al. 1982; Morley and Shafer 1982; Chopra et al. 1990), and elevated TSH concentrations (Chopra et al. 1990). Studies of discrete psychiatric illnesses have yielded different patterns of HPT function.

The majority of depressed adults have thyroid function tests that fall within the normal range, though decreases versus normals have been reported (Joffe et al. 1984; Orsulak et al. 1985; Loosen 1987; Prange et al. 1987; Rubin et al. 1987).

Our finding of elevated T3, T4, and TSH stands in contrast to the majority, but not all, of studies of depressed patients by a subtle elevation, rather than reduction, in HPT status. The elevation does not appear to be due to altered peripheral conversion or production of thyroid hormones. Increased basal HPT status would provide a mechanism to explain the significant blunting of the TSH response to TRH in two adult studies Aizenberg et al. 1991; Lucey et al. 1993. Due to the significant elevation of TSH versus controls, we conclude that the HPT increases are mediated at a level above the pituitary (presumably associated with chronically increased TRH secretion) and may involve neurotransmitters such as serotonin (5-HT), and perhaps dopamine, both of which have known effects on HPT function (Tuomisto and Mannisto 1985), and have also been implicated in the pathogenesis of OCD (Murphy et al. 1989). It is likely that our use of the ultrasensitive IRMA assay for TSH enabled the detection of increased TSH in our OCD sample not seen in prior reports, in spite of all values falling within the normal range for TSH.

While some reports have noted decreases in T3 induced by clomipramine in depressed adult patients (Schlienger et al. 1981) and in rats (Massol et al. 1990), to our knowledge this is the first description of the effects of clomipramine on thyroid indices in patients with obsessive-compulsive disorder (OCD).

Our data demonstrated that chronic administration of clomipramine, an antidepressant and antiobsessional medication with prominent effects on serotoninergic neurotransmission, did produce significant effects on circulating measures of thyroid function in this sample of children and adolescents with OCD. Our findings largely confirm other reports that have examined thyroid function changes in depressed adults receiving antidepressant treatment, including clomipramine and desipramine (Schlienger et al. 1980; Brady and Anton 1989). Our data and those of others is mostly in agreement with animal studies that have noted reduced thyroid hormones (Massol et al. 1990) and TSH (Mueller et al. 1976) following chronic treatment with tricyclic antidepressants, including clomipramine (Massol et al. 1990). However, these effects are also somewhat selective, in that a reduction in T3 and TSH was observed in the absence of a significant change in T4. We believe that these data, taken together with recent preclinical data and the exploratory correlations between baseline HPT status and treatment response (see below), have possible implications for the understanding of the augmenting effects of exogenous thyroid hormones in the treatment of depression, and conversely, for the absence of a therapeutic benefit of adjunctive T3 in combination with clomipramine for partially responding patients with OCD (Pigott et al. 1991).

Interestingly, we observed that both $\mathrm{T} 4$ and TSH concentrations at baseline were highly correlated with the percent symptom reduction at 8 weeks of treatment as measured by the CY-BOCS. While this finding is tempered by a modest sample size, absence of double-blind treatment, and not correcting for multiple tests, it stands in agreement with the notion that elevated HPT status is associated with a greater 
degree of biological dysregulation at a level above the pituitary, which itself may be related to the underlying pathophysiology of OCD.

Although the reciprocal relations between brain 5-HT systems and the HPT axis are complex, available information suggests that alterations in thyroid status are likely to produce effects on 5-HT neurotransmission, and conversely, that experimental manipulations of 5-HT can influence HPT measures (Henley et al. 1991; Sandrini et al. 1991).

We would propose that treatment-refractory patients may benefit from interventions that reduce HPT status, such as propylthiouracil (PTU). This conclusion is certainly speculative, but is consistent with the above mentioned study finding no effect of T3 augmentation of clomipramine.

\section{CONCLUSION}

Taken together, our data and the earlier work of others on thyroid function in OCD support the pursuit of larger and more comprehensive studies of the frequency of HPT axis differences in OCD patients as well as the relation of baseline thyroid state to treatment response in OCD and the effects of antiobsessional agents on HPT regulation. The fact that significant differences were observed in children and adolescents with OCD should also strongly encourage other studies of younger patients with the disorder, a group that to date has unfortunately received less attention.

\section{ACKNOWLEDGMENTS}

This work was supported, in part, by the National Institutes of Mental Health grant MH00722 (JTM). The technical assistance of Mary Price is greatly appreciated.

\section{REFERENCES}

Aizenberg D, Hermesh H, Gilad I, Munitz, Tyano S, Laron Z, Weizman A: TRH stimulation test in obsessive-complusive patients. Psychiatry Res 38: 21-26, 1991.
American Psychiatric Association: Diagnostic and Statistical Manual, 3rd ed revised (DSM-III-R). American Psychiatric Association, Washington, D.C. 1987.

Brady KT, Anton RF: The thyroid axis and desipramine treatment in depression. Biol Psychiatry 25:703-709, 1989.

Caplan RH, Pagliara AS, Wickus G, Goodlund LS: Elevation of the free thyroxine index in psychiatric patients. I Psychiatric Research 17:267-274, 1982/1983.

Chopra IJ, Solomon DH, Huang T-S: Serum thyrotropin in hospitalized psychiatric patients: Evidence for hyperthyrotropinemia as measured by an ultrasensitive thyrotropin assay. Metabolism 39:538-543, 1990.

Cohen KL, Swigar ME: Thyroid function screening in psychiatric patients. JAMA 242:254-257, 1979.

Gold MS, Goodwin FK, Wehr TA: Pituitary thyrotropin response to thyrotropin-releasing hormone in affective illness: Relationship to spinal fluid amine metabolism. Am J Psychiatry 134: 1028-1031, 1977.

Goodman WK, Price LH: The Yale-Brown Obsessive Compulsive Scale I. Development, use, reliability. Arch Gen Psychiatry 46:36-44, 1989.

Hanna GL, McCracken JT, Cantwell DP: Prolactin in childhood obsessive-compulsive disorder: Clinical correlates and response to clomipramine. I Am Acad Child Adoles Psychiatry 30:173-178, 1991.

Henley WN, Chen X, Klettner C, Bellush LL, Notestine MA: Hypothyroidism increases serotonin turnover and sympathetic activity in the adult rat. Can J Physiol Pharmacol 69:205-210, 1991.

Joffe RT, Levitt AJ: Major depression and subclinical (Grade 2) hypothyroidism. Psychoneuroendocrinology 17:215-221, 1992.

Joffe RT, Roy-Byrne PP, Uhde TW, Post RM: Thyroid function and affective illness: A reappraisal. Biol Psychiatry 19:1685-1691, 1984.

Joffe RT, Swinson RP: Thyroid function in obsessive-compulsive disorder. Psychiatric I Univ Ottawa 13:215-216, 1988.

Linnoila M, Gold P, Potter WZ, Wehr TA: Tricyclic antidepressants do not alter thyroid hormone levels in patients suffering from a major affective disorder. Psychiatry Res 4:357-360, 1981.

Loosen PT: Thyroid hormones and affective state. In Halbreich U (ed), Hormones and Depression. Edited by Halbreich U. New York, Raven Press, 1987, pp. 357-383.

Lucey JV, Butcher G, Clare AW, Dinan TG. The anterior pituitary responds normally to protirelin in obsessive-compulsive disorder: evidence to support a neuroendocrine serotonergic deficit. Acta Psychiatr Scand 87:384-388, 1993.

Massol J, Martin P, Chatelain F, Puech AJ: Tricyclic antidepressants, thyroid function, and their rela- 
tionship with the behavioral responses in rats. Biol Psychiatry 28:967-978, 1990.

Morley JE, Shafer RB: Thyroid function screening in new psychiatric admissions. Arch Intern Med 142:591-593, 1982.

Mueller GP, Twohy CP, Chen HT, Advis JP, Meites J: Effects of L-trytophan and restraint stress on hypothalamic and brain serotonin turnover, and pituitary TSH and prolactin release in the rat. Life Sciences 18:715-718, 1976.

Murphy DL, Zohar J, Benkelfat MT, Pato MT, Pigott TA, Insel TR: Obsessive-compulsive disorder as a 5-HT subsystem-related behavioral disorder. $\mathrm{Br}$ Psychiatry 155:15-24, 1989.

Nordgren L, Scheele C: Nortriptyline and pituitarythyroid function in affective disorder. Pharmacopsychiaty 14:61-65, 1981.

Noshirvani HF, Kasvikis Y, Marks IM, Tsakiris F, Monteiro WO: Gender-divergent aetiologic factors in obsessive-compulsive disorder. Br J Psychiatry 158:260-263, 1991.

Orsulak PJ, Crowley G, Schlesser MA, Giles D, Fairchild C, Rush AJ: Free triiodothyronine (T3) and thyroxine (T4) in a group of unipolar depressed patients and normal subjects. Biol Psychiatry 20:1047-1054, 1985.

Pigott TA, Pato MT, L'Heureux F, Hill JL, Grover GN, Bernstein SE, Murphy DL: A controlled comparison of adjuvant lithium carbonate or thyroid hormone in clomipramine-treated patients with obsessive-compulsive disorder. I Clin Psychopharmacol 11:242-248, 1991.

Rubin RT, Poland RE, Lesser IM, Martin DJ: Neuroendocrine aspects of primary endogenous depression-IV. Pituitary-thyroid axis activity in patients and matched control subjects. Psychoneuroendocrinology 12:333-347, 1987.

Sandrini M, Marrama D, Vergoni AV, Bertolini A: Effects of thyroid status on the characteristics of alpha1-, alpha2-, beta, imipramine, and GABA receptors in the rat brain. Life Sciences 48:659666, 1991.

Schlienger JL, Kapfer MT, Singer L, Stephan F: The action of clomipramine on thyroid function. Horm Metab Res 12:481-482, 1980.

Spratt DI, Pont A, Miller MB, McDougall IR, Bayer MF, McLaughlin WT: Hyperthyroxinemia in patients with acute psychiatric disorders. Am J Med 73:41-48, 1982.

Tuomisto J, Mannisto P: Neurotransmitter regulation of anterior pituitary hormones. Pharmacol Rev 37:249-332, 1985.

Address reprint requests to: James T. McCracken

UCLA Neuropsychiatric Institute 760 Westwood Plaza Los Angeles, CA, 90024-1759

E-mail: JMcCracken@mednet.ucla.edu 